\title{
Feedback no processo de aprendizagem: percepção dos estudantes de Odontologia em uma universidade brasileira
}

Cynthia Moura Louzada Farias*; Brisa Daniele Furtado Cardoso**; Edson Theodoro dos Santos Neto***; Raquel Baroni de Carvalho****; Donald Curtis*****

* Mestranda em Odontologia do Programa de PósGraduação em Clínica Odontológica da UFES

** Graduanda do Curso de Odontologia UFES

*** Professor Adjunto do Departamento de Medicina Social da UFES

**** Professora Associada do Curso de Odontologia da UFES

***** Professor do Curso de Odontologia da Universidade da Califórnia, São Francisco/CA, EUA.

\section{RESUMO}

O objetivo do estudo foi analisar a opinião dos estudantes do ciclo profissionalizante do curso de Odontologia da Universidade Federal do Espírito Santo (UFES) e discutir a respeito de assuntos que envolvem a prática do feedback no processo ensino-aprendizagem. Trata-se de um estudo exploratório, de abordagem qualitativa, no qual foram aplicados questionários semiestruturado e entrevista aos alunos do $7^{\circ}$ ao $10^{\circ}$ período do curso de odontologia. Os dados revelaram que os alunos tendem a valorizar feedback, afirmando ser importante para sua aprendizagem e veem como crítica construtiva a correção de suas atividades, embora admitam que a sua eficácia dependa de clareza com que é feita, bem como da relação que os alunos mantêm com o professor. Concluiu-se que o feedback é uma parte essencial da educação universitária. Os professores precisam estar envolvidos no processo de repaginação do ensino e comprometidos em conhecer e utilizar novas ferramentas que contribuam para a busca do conhecimento e a formação profissional do aluno.

Descritores: Ensino superior. Formação profissional. Retroalimentação. Aprendizagem.

\section{INTRODUÇÃO}

O grande desafio das instituições de ensino superior é formar profissionais com o perfil que atenda às diversas necessidades do mercado de trabalho. $\mathrm{O}$ professor de odontologia precisa estar atento à reconstrução do modelo de formação do cirurgião-dentista, dominando os métodos mais eficazes para alcançar os objetivos dessa prática a fim de melhorar a experiência de aprendizagem dos alunos $^{1}$.

A busca por metodologias inovadoras a serem utilizadas nas práticas de sala de aula refletem, muitas vezes, o insucesso, pois não se priorizam as reais necessidades e interesses dos alunos. É pertinente que os saberes sociais que se manifestam na prática docente e que são apresentados aos alunos, sejam sempre analisados e o ideal é que os saberes docentes caminhem para buscar atender às necessidades discentes, pois é a partir de práticas que respeitem as necessidades singulares que se busca demonstrar a importância e o sentido que a escola possui ${ }^{2}$.

Estudos mostram que o feedback tem se 
mostrado uma ferramenta eficaz utilizada na prática de obtenção de melhor desempenho e como uma parte essencial da educação universitária $^{3,4}$. Ele ajuda os alunos a ampliar o seu potencial de conhecimento em diferentes estágios de formação, aumentar a sua consciência dos pontos fortes e áreas de melhoria, e identificar ações a serem tomadas para melhorar o desempenho durante e após o seu processo de formação ${ }^{3,4}$. Ao longo dos últimos anos, a busca por novas maneiras que potencializem a aprendizagem está iniciando nos cursos da área de saúde ${ }^{4}$.

No comportamento em sala de aula e na prática clínica, as atitudes dos futuros profissionais são regulares e rotineiramente observadas e, por meio de uma série de critérios, pode-se verificar as necessidades em relação à lapidar a forma de abordagem ou resolução dos procedimentos executados. Assim, o mero pensar precisa ser substituído pelo saber pensar e, sobretudo, pelo aprender a aprender pensar e fazer 5 .

O feedback promove a melhor aprendizagem, informando o progresso do aluno ou a falta dele, aconselhando-o sobre as necessidades observadas e os recursos disponíveis para facilitar a sua aprendizagem, além de motivá-los ao envolvimento na execução das atividades ${ }^{6}$. É uma ferramenta potencializadora da aprendizagem, e não é orientada para medir os conhecimentos demonstrados pelos alunos ou as suas habilidades específicas. Isso permite a diminuição do reducionismo que se apresenta nas insuficiências das teorias de aprendizagem no ensino superior ${ }^{1,6}$.

No passado, o feedback era visto apenas como algo que determinava um grau de avaliação, atualmente ele é encarado também como uma ferramenta para a aprendizagem. Além disso, guia a aprendizagem do aluno, dado que define aquilo que o estudante considera importante, bem como o tempo que despende ao realizar as tarefas propostas, é determinante para sua motivação, estimulandoo para dar o seu melhor. Isso tem colocado grande ênfase na compreensão dos processos por meio dos quais a prática do feedback interage para influenciar o desenvolvimento de formação profissional $^{1,7}$.

A compreensão de alguns princípios básicos sobre a aprendizagem ${ }^{8}$, pode auxiliar o professor na prática de melhores maneiras de agir no dia a dia. Esses são conhecidos como os princípios FAIR: fornecer feedback para o aluno, envolver o aluno na aprendizagem ativa, individualizar a aprendizagem às necessidades pessoais do aluno e tornar o aprendizado relevante. A aplicação desses princípios, na afirmativa dos autores, pode levar a uma aprendizagem mais eficaz, permitindo que um professor mediano se torne um bom professor e que o bom professor se torne um excelente professor.

Para implementar estratégias inovadoras, essa prática deverá ser planejada dentro de um grau de amplitude que garanta sua interação ao nível de reflexão e interação com as propostas curriculares em que se atua, dentro do esquema "pensar-agir-pensar", preservando o caráter formativo da educação em saúde ${ }^{9}$.

Por isso, o feedback é fundamental para o desenvolvimento da competência e confiança dos alunos em todas as fases de sua carreira. Ao fazê-lo, o professor deve se concentrar na ação que se observa, e não no aluno; as pontuações devem ser específicas e relacionadas com os objetivos pessoais do aluno, para que possam ajudar a reforçar o desempenho desejável e corrigir o mau desempenho. As maneiras de se realizar um feedback eficaz estão associadas ao ensino de alta qualidade, na percepção dos estudantes ${ }^{10}$.

Dessa forma, o objetivo do estudo foi analisar a opinião dos estudantes do ciclo 
profissionalizante do curso de Odontologia da UFES a respeito de assuntos que envolvem a prática do feedback no processo ensinoaprendizagem, como eles veem essas práticas, bem como a importância dessa ferramenta como facilitadora de sua aprendizagem.

\section{METODOLOGIA}

O presente estudo está aninhado em um projeto exploratório descritivo mais amplo, realizado no âmbito do Programa de Pósgraduação em Clínica Odontológica na Universidade Federal do Espírito Santo (UFES), e foi realizado no período de agosto a dezembro de 2014. A pesquisa envolveu alunos e professores do curso de Odontologia da UFES que, à época da coleta de dados, possuía aproximadamente 260 alunos matriculados e 70 docentes em atuação. O curso está estruturado em dez períodos (com duração de cinco anos), após passar por uma mudança no currículo, de acordo com as Diretrizes Curriculares Nacionais $(\mathrm{DCN})^{11}$ de 2002. A pesquisa foi aprovada pelo Comitê de Ética em Seres Humanos do Centro de Ciências da Saúde da UFES sob o número de registro CAAE 339550144.0000 .5060 .

Os sujeitos analisados foram os alunos do $7^{\circ}$ ao $10^{\circ}$ período do curso (120 alunos que estavam em atividade clínica), matriculados no período de 2014/2. O critério de inclusão para participação da primeira etapa da pesquisa (aplicação de questionário), foi estar matriculado em 2014/2 em disciplinas do $7^{\circ}$ ao $10^{\circ}$ períodos. Foram excluídos todos os outros alunos que não se encontravam nessa condição. O questionário semiestruturado corresponde à parte quantitativa da pesquisa e foi respondido por 103 participantes.

A entrevista corresponde à parte qualitativa da pesquisa. Para essa etapa, os participantes foram selecionados dentre os alunos que responderam o questionário, e o critério de saturação foi utilizado para estabelecer o número de entrevistados, de maneira que, à medida que se verificou que as respostas não estavam acrescentando percepções diferentes à pesquisa, encerramos a atividade. Foram entrevistados 20 alunos, os quais foram identificados em suas falas pela letra A (aluno), seguida do número correspondente à ordem cronológica da entrevista e então ao período em que o estudante estava matriculado. Assim, A4(10) representa a quarta entrevista do grupo de alunos do $10^{\circ}$. período.

Os dois instrumentos foram organizados e adaptados dos instrumentos de Lazzarin ${ }^{12}$, Araújo; Mello ${ }^{13}$ e Castro $\mathrm{Jr}^{14}$. Os instrumentos foram validados por um questionário piloto aplicado em outros cursos de odontologia do estado. Após a validação, houve um contato prévio individual com os possíveis entrevistados, para explicar os objetivos da investigação e solicitar sua colaboração. Os dados foram submetidos à análise descritiva e a entrevista gravada e transcrita, submetida à análise temática de conteúdo ${ }^{15}$, com o objetivo de conhecer e descrever como os alunos vêem as práticas de feedback, bem como a importância da ferramenta como facilitadora de sua aprendizagem, no curso de odontologia da UFES.

\section{RESULTADOS E DISCUSSÃO}

A maior parte dos estudantes entrevistados é do sexo feminino (aproximadamente $76 \%$ ) e está matriculada no $9^{\circ}$ período $(40 \%)$. Em relação às experiências de aprendizagens mais eficazes para os alunos, destaca-se a atividade clínica $(43,8 \%)$. Ao serem questionados na entrevista em relação à feedback e aprendizagem, os participantes afirmaram que é muito importante para a sua aprendizagem discutir ideias com os professores e receber constantes orientações 
sobre suas ações.

Dados do questionário permitiram analisar o perfil dos estudantes e por meio da interpretação das narrativas, fazer sua relação com o referencial teórico utilizado. Aspectos importantes do perfil do professor que dá o feedback e do destinatário incluem diferenças de sexo, idade ou formação educacional e cultural. Os alunos diferem entre si em relação às atitudes, hábitos, práticas, disposição para realizar as suas atividades, cumprimento de prazos, presteza, interesse, percepção de valores, postura diante dos colegas e pacientes e em uma série infindável de outros fatores. A otimização dos resultados obtidos no desempenho deles pressupõe permanente interação entre as partes envolvidas, professores e alunos, nas ações do dia a dia, permitindo que haja uma livre troca de informações, de avaliações críticas e correções de rumos, quando necessárias ${ }^{16}$.

Estudantes do sexo feminino relatam receber melhor o feedback ${ }^{7}$. Esse fato não é necessariamente um obstáculo, mas pode tornar o feedback um ato tenso e desmotivador, se não for observado. Pode também existir uma série de barreiras para dar um feedback eficaz no contexto da educação em saúde, como o medo de prejudicar o aluno ou a relação alunoprofessor, deixando o aluno resistente ou defensivo quando receber críticas ${ }^{8}$. Estudos acadêmicos mostram que ofeedback deve se ser dado por meio de orientações sobre como corrigir o comportamento, com a consistência de várias fontes de argumentos, para não haver uma falta de respeito para com quem está dando o feedback ao avaliar. Sempre que possível, o feedback deve ocorrer logo após a situação observada ou a tarefa desempenhada. Concentrar-se no positivo é a regra básica, especialmente no feedback mais negativo ${ }^{17-20}$.

Entretanto, não há como informar ao aluno que a sua hipótese diagnóstica ou sua conduta e resposta estavam erradas, ou ainda, que ele não observou os detalhes necessários e nem colheu todos os dados durante a história clínica, sem causar uma sensação de desapontamento ou frustração. Por outro lado, essa informação é essencial e não pode ser omitida. A aprendizagem pode ser aprimorada melhorando as habilidades do aluno através de um feedback ${ }^{21}$.

Faz-se necessário lembrar que o docente que orienta as tarefas propostas necessita conhecer tecnicamente os temas ou questões a ele relacionados. Não é imperativo, porém, que o mesmo seja especialista nessa área. Contudo, é desejável que seu conhecimento sobre o tema seja suficiente para permitir um redirecionamento em casos nos quais as discussões se desviam do eixo proposto. $\mathrm{O}$ papel deste docente é intervir na construção de hipóteses ou nos tópicos de aprendizagem que estão sendo levantados pelo aluno, estimulando-o a refletir criticamente sobre a melhor solução para o procedimento ${ }^{17}$.

Um modelo para dar feedback de forma eficaz em ambientes clínicos foi desenvolvido por Pendleton $(2003)^{7}$, que orienta conhecer o aluno, observar se o mesmo quer e/ou está pronto para receber o feedback. $\mathrm{O}$ autor recomenda deixar que o aluno faça comentários a respeito do procedimento executado. $\mathrm{O}$ professor pode concordar que este foi bem feito, mas deve dizer como pode ser melhorado. A partir daí ambos elaboram um plano de ação. A partir desse ponto percebe-se que feedback pode ser usado para promover a reflexão, especialmente quando é corretivo e discordante da auto percepção, o que oferece ao aluno uma oportunidade para o pensamento crítico ${ }^{6,14,18}$. Os alunos entrevistados disseram que consideram importante receber o feedback dos professores para o desenvolvimento da sua aprendizagem, mas relataram que este acontece muito pouco no curso de odontologia: 
"[...]eu acho importante, acho que isso demonstra respeito e interesse pelo aluno. Acho que poucos fazem isso. A1(10); Com certeza, é importante, mas só acontece às vezes. A2(10); [...] ajuda muito porque nós precisamos aprender, $e$ aprender certo. Eu acho que na verdade são poucos que fazem [...]" A4(10).

As afirmativas dos alunos em relação à importância dessa prática são fundamentadas por vários autores $6,18,22,23$, os quais identificaram o feedback como uma parte vital do ensino-aprendizagem no processo de formação profissional. Os professores que utilizam o feedback são mais eficazes em motivar os alunos no ensino superior, em particular, a aprender.

Inicialmente, esta forma diferente de trabalho pode ser mais difícil para os professores do que usar a abordagem didática tradicional, mas a prática no uso deste método oferece suporte à sua eficácia em ambientes clínicos. É necessário estar atento para o fato de que o feedback é parte do diálogo ou do processo de interação e troca entre professor e aluno, não uma comunicação de mão única. Muitas situações clínicas no ensino em odontologia envolvem a integração de conhecimentos, habilidades e comportamentos em ambientes complexos e muitas vezes estressantes, com limitação de tempo e muito serviço, o que gera pressões sobre o professor e o aluno ${ }^{3,5,19}$.

O feedback ajuda desenvolver o diálogo entre o aluno e o professor. É um modo eficaz de iniciar a reflexão e contribuir para a formação de profissionais autônomos. ou seja, capazes de tomar decisões embasadas em observações e resultados, bem como professores conscientes do próprio aperfeiçoamento profissional. Baseia-se na auto avaliação dos alunos, num processo de colaboração e ajuda para assumirem a responsabilidade por sua própria aprendizagem ${ }^{24}$. Alguns alunos entrevistados ressaltaram que a relação professor-aluno pode influenciar esse feedback e, consequentemente, a sua aprendizagem:

"[...] quando o professor conhece e sente que o aluno realmente está interessado ele parece que demonstra mais interesse que a gente aprenda. Ai ele chega para ajudar, então você sabe que ele está dando seu melhor, para eu melhorar A2(10) [...] depende do meu desempenho, da minha vontade de querer aprender, aí o professor se interessa em me ensinar, dar atenção no que eu preciso [...] A4(10) [...] eu acho que para aprender melhor envolve muito o relacionamento pessoal dele (professor) com os alunos (...) se ele também dá uma certa abertura pra perguntar, pra discutir [...]." A1(10).

A prática de feedback no ensino em saúde é uma ferramenta potencializadora da aprendizagem e não deve ser orientada para tomar medidas sobre os conhecimentos e as habilidades específicas do aluno. Deve ser focada em comportamentos que podem ser alterados e não em traços de personalidade. Ser claro a respeito do feedback é essencial. O professor deve estar focado no desenvolvimento profissional global do aluno ou aos resultados dos procedimentos e objetivos pretendidos nas atividades. Não é um exagero descrever o feedback como "o combustivel que impulsiona um melhor desempenho no processo de formação profissional" ${ }^{16,25}$. Deve ser fornecido com sensibilidade e de forma adequada, sendo fácil, especialmente se este professor já trabalha com aquele aluno há algum tempo 21,25,26 $^{2}$

Os alunos afirmam que, depois de terminarem uma tarefa, esperam que o professor os informe acerca da mesma e declaram que é importante para o desenvolvimento da sua aprendizagem discutir ideias com os 
professores:

“[...] o professor precisa chegar, falar o nosso erro e ensinar, aí você aprende com o que errou. Ele deve conseguir repassar isso, dentro do que você fez, ali, na prática. [...] A3(7) [...] pra mim é importante o professor preocupar se você está conseguindo fazer o trabalho na prática, porque a prática exige um conhecimento muito maior, e exige técnica [...] é bom quando ele tem métodos pra melhorar a aprendizagem, é tudo tão corrido que às não dá tempo. A4(7) [...] além de ser um bom professor na teoria, ele tem que ter a facilidade de passar o que ele sabe e como se faz, quando a gente precisa [...]" A5(7).

Além de valorizar as informações transmitidas pelos docentes quando terminam sua tarefa, os participantes da pesquisa consideram o feedback um elemento importante para a sua aprendizagem. Por outro lado, os dados apontam para a dificuldade em realizar tarefas sem receberem esse feedback, sentindo-se mais inseguros, corroborando a importância da comunicação e do diálogo no processo ${ }^{9}$.

Muitas situações clínicas envolvem a integração de conhecimentos, habilidades e comportamentos, em experiências novas, ambientes complexos e muitas vezes estressantes, com limitação de tempo e muito serviço, o que gera pressões sobre o professor e o aluno ${ }^{3,18,21}$.

Além disso, os professores que exercem a docência em atividades clínicas, como acontece no curso de odontologia, muitas vezes observam situações em que precisam intervir para melhor contribuir para a solução de um caso, mas hesitam em ensinar sobre ele, pela falta de tempo para isso. Glick et al. (2009) ${ }^{27}$ mostraram o desenvolvimento de estratégias eficazes de tempo para o ensino em saúde. Usaram vários cenários para ilustrar os principais componentes do modelo: diagnosticar o aluno, ensinar rapidamente a necessidade requerida pelo aluno e fornecer feedback ao aluno. O modelo é simples, fácil de usar e permite a incorporação no exercício da prática do ensino clínico em saúde, numa troca permanente de conhecimento e informações ${ }^{27}$.

\section{CONCLUSÃO}

Partindo do pressuposto de que o ensino odontológico precisa estar adequado às recomendações sugeridas nas DCN, é discutido no âmbito acadêmico que ele deva ser repaginado, para que os cirurgiões-dentistas desenvolvam competências e habilidades críticas e reflexivas em suas práticas durante o exercício profissional. O feedback é apontado como um instrumento de ensino no curso de medicina, portanto, faz-se necessário trabalhos que o avaliem no curso de odontologia.

É pertinente que os professores estejam comprometidos a colocar em prática, independentemente de sua área específica de atuação, as ferramentas que auxiliam a melhor performance profissional dos alunos. Devem transmitir segurança ao aluno, de modo que este possa realizar seu aprendizado de maneira consciente e reflexiva, sabendo quais dificuldades poderá encontrar e, sobretudo, sabendo quais recursos estão disponíveis para superá-las.

\section{REFERÊNCIAS}

1. Zeferino AMB, Domingues RCL, Amaral E. Feedback como estratégia de aprendizado no ensino médico. Rev Bras Educ Med 2007; 31(2):176-9.

2. Quadros C, Azambuja G. Saberes e dizeres sobre formação de professores na UNIFRA. Santa Maria: Centro Universitário Franciscano, 2003.

3. Ramsden P. Learning to teach in higher 
education. London: Routledge; 1992.

4. Schmidt RA. Aprendizagem e performance motora: dos princípios à prática. São Paulo: Movimento; 1993.

5. Bordenave J, Pereira A. A estratégia de ensino aprendizagem. 26a ed. Petrópolis: Vozes; 2005.

6. Gipps C. Socio-cultural aspect of assessment. Rev Educ Res 1999; 24:355-92.

7. Pendleton D, Schofield T, Tate P, Havelock P. The consultation: an approach to learning and teaching. Oxford: Oxford University Press; 2003.

8. Harden RM, Laidlaw JM. Essential skills for a medical teacher - an introduction to teaching and learning in medicine. Edinburgh: Churchill Livingstone; 2014.

9. Higgins R, Hartley P, Skelton A. Getting the message across: the problem of communicating assessment feedback. Teaching Higher Educ 2001; 6(2):269-74.

10. Gordon J. ABC of learning and teaching in medicine: one to one teaching and feedback. BMJ 2003; 326(7388):543-5.

11. Brasil. Resolução CNE/CES 3/2002. Diário Oficial da União, Brasília, 4 de março de 2002. Seção 1, p.10. Disponível em: http://portal. mec.gov.br/cne/arquivo/pdf/CES32002.pdf

12. Lazzarin HC, Nakama L, Cordoni LJr. O papel do professor no processo ensinoaprendizagem: percepção de professores e alunos de odontologia. Londrina, 2005. Dissertação (Mestrado em Saúde Coletiva). Universidade Estadual de Londrina.

13. Araújo RPC, Mello SMF. Cursos de graduação em Odontologia: a formação docente Pesqui Bras Odontopediatria Clín Integr 2011; 11(4):615-25.

14. Castro R Jr. Educação Superior: os saberes pedagógicos do bacharel docente. Brasília, 2008. Dissertação (Mestrado em Educação). Universidade de Brasília.

15. Bardin L. Análise de conteúdo. Lisboa: Edições 70; 2009.

16. Sargeant J, Mann K, Van Der Vleuten C, Metsemakers J. Reflection: a link between receiving and using assessment feedback. Adv Health Sci Educ 2009; 14(3):399=410.
Disponível em: http://www.springerlink. com/content/55h76108ru421647

17. Bochner D, Badovinac RL, Howell TH, Karimbux NY. Tutoring in a problem- based curriculum: expert versus nonexpert. J Dent Educ 2002; 66:1246-51.

18. Ende J. Feedback in clinical medical education. J Am Med Assoc 1983; 250(6):777-81.

19. Eva KWE, Heather A, Lockyer EHJ, Loney E, Mann K, Sargeant J. Factors influencing responsiveness to feedback: on the interplay between fear, confidence, and reasoning processes. Adv Health Sci Educ 2012; 17:1526.

20. Vassilas C, Ho L. Video for teaching purposes. Adv Psychiatric Treat 2000; 6:304-11.

21. Irby DM. Teaching and learning in ambulatory care settings: a thematic review of the literature. Acad Med 1995; 70:898-931.

22. Norcini J, Mckinley, D. Assessment methods in medical education. Teach Edu 2007; 23:23950 .

23. Troncon L. Avaliação do estudante de medicina. Medicina 1996; 29(4):430-9.

24. Pereira DR, Flores, MA. Avaliação e feedback no ensino superior: um estudio na Universidade do Minho. RIES 2013;10:40-54. Disponível em: http://ries.universia.net/index.php/ries/ article/view/257

25. Parsloe E. Coaching, mentoring and assessing: a practical guide in developing competence. London: Nichols Publishing; 1995.

26. Kurtz S, Silverman J, Draper J. Teaching and learning communication skills in medicine. Oxford: Radcliffe Medical Press; 1998.

27. Glick SB, Fernandez L, Irby DM, Harleman E, Fernandez A. Teaching about health care disparities in the clinical setting. J Gen Intern Med 2009; 25(Suppl 2):95-101. Disponível em: http://springer.com/article/10.1007/s116 06-009-1203-6.

\section{ABSTRACT}

Feedback in the learning process: perceptions among dental students at a Brazilian

\section{University}

This study aimed at analyzing opinions among 
students in the professionalizing cycle of the Dentistry Program at Federal University of Espírito Santo (UFES), Brazil, and discussing questions regarding feedback in the teachinglearning process. It is a qualitative exploratory study that adopted semi-structured questionnaires and interviews with $1037^{\text {th }}$ and $10^{\text {th }}$ term dental students. The data show that students tend to value feedback, which they see as constructive criticism to correct their activities, even though they admit that feedback efficacy and use depend on understanding the tasks, as well as on the relationship students have with professors. We concluded that feedback is an essential part of university education.
Professors need to be involved in continued education processes and be committed to understanding and using new tools that contribute to students' pursuit of knowledge and professional training.

Descriptors: Higher education. Professional training. Feedback. Learning.

Correspondência para:

Cynthia Moura Louzada Farias e-mail: cynthialousada1 @ hotmail.com Av. Marechal Campos, 1468 Maruipe 29043-900 Vitória/ES 